Case Report

\title{
Evaluation of Teriparatide for Treatment of Osteoporosis in Four Patients with Cystic Fibrosis: A Case Series
}

\author{
Oranan Siwamogsatham, ${ }^{1,2}$ Kelly Stephens, ${ }^{2}$ and Vin Tangpricha ${ }^{2}$ \\ ${ }^{1}$ Department of Pediatrics, Samitivej Srinakarin Hospital, Bangkok Hospital Group, 488 Srinakarin Road, Suanluang, \\ Bangkok 10250, Thailand \\ ${ }^{2}$ Division of Endocrinology, Metabolism and Lipids, Department of Medicine, Emory University School of Medicine, \\ 101 Woodruff Circle NE, WMRB 1301, Atlanta, GA 30322, USA
}

Correspondence should be addressed to Oranan Siwamogsatham; oranan.w@gmail.com

Received 24 December 2013; Accepted 3 February 2014; Published 6 March 2014

Academic Editors: K. Iida, M. P. Kane, and W. V. Moore

Copyright (C) 2014 Oranan Siwamogsatham et al. This is an open access article distributed under the Creative Commons Attribution License, which permits unrestricted use, distribution, and reproduction in any medium, provided the original work is properly cited.

Introduction. Bone disease is a common complication of cystic fibrosis (CF). To date, there have been no reports on the effectiveness of teriparatide, recombinant human parathyroid hormone, to treat CF-related bone disease. Case Presentation. We report on four patients with CF-related bone disease who were treated with teriparatide. Three patients completed two years of therapy with teriparatide, and all had significant improvements in their bone mineral density (BMD). One patient was unable to tolerate teriparatide and discontinued treatment 1 week into therapy. Conclusion. Teriparatide may be a potential treatment option for CF-related bone disease. This report highlights the need for further investigation into the use of teripartide in the CF population.

\section{Introduction}

Cystic Fibrosis (CF), caused by mutations in the cystic fibrosis transmembrane conductance regulator (CFTR) protein, is a common lethal genetic disease among Caucasians. As survival has improved, new complications from CF have emerged, including CF-related bone disease [1-3]. The major contributing factors to bone disease in CF include vitamins $\mathrm{D}$ and $\mathrm{K}$ malabsorption, poor nutritional status, physical inactivity, chronic inflammation, glucocorticoid therapy, delayed puberty, and hypogonadism [4-6]. These factors result in decreased bone mineral density (BMD), osteopenia, osteoporosis, fragility fractures, and kyphosis, which can cause significant morbidity and potential exclusion from lung transplantation candidacy [7]. Bisphosphonates, antiresorptive agents, have been shown to be efficacious in treating CF-related bone disease and currently are the mainstay of treatment. Studies on the use of teriparatide, recombinant human parathyroid hormone, in CF-related bone disease have not been reported to date.

\section{Case Presentation}

We report on four patients with CF-related bone disease who were treated with teriparatide $20 \mathrm{mcg}$ subcutaneously once a day for a two-year period at the Emory University Cystic Fibrosis clinic. The study was approved by IRB at Emory University and all patients provided written consent for presenting their data. Demographic characteristics of our patients are displayed in Table 1.

Case 1. Patient 1 was a 59-year-old Caucasian female with a two-year history of CF who was referred to our clinic for evaluation of osteopenia. She was diagnosed with adult onset CF based on clinical findings after she developed a pulmonary mycobacterium avium complex (MAC) infection that was very difficult to clear. She reported being treated with prednisone two times in the past for CF exacerbations. Since menopause at 52 years of age, she had been treated with raloxifene for osteoporosis prevention. She also was on daily calcium and vitamin D supplementation with $1,200 \mathrm{mg}$ 
TABLE 1: Demographic data.

\begin{tabular}{|c|c|c|c|c|}
\hline & Patient 1 & Patient 2 & Patient 3 & Patient 4 \\
\hline Age (year) & 59 & 35 & 31 & 48 \\
\hline BMI $\left(\mathrm{kg} / \mathrm{m}^{2}\right)$ & 19.6 & 21.8 & 20 & 18.5 \\
\hline Ethnicity & Caucasian & Caucasian & Caucasian & Caucasian \\
\hline Gender & Female & Female & Male & Female \\
\hline Gene mutation & - & $\Delta$ F508 Homozygous & 2184delA/R553 & R117H/R560T \\
\hline Baseline $25(\mathrm{OH}) \mathrm{D}(\mathrm{ng} / \mathrm{mL})$ & 48 & 56 & 33 & 47 \\
\hline \multicolumn{5}{|l|}{ BMD (T-score) } \\
\hline L1-L4 & -1.6 & $-1.6^{*}$ & -2.1 & -0.7 \\
\hline Left femoral neck & -2.2 & $-1.9^{*}$ & -1.9 & -1.5 \\
\hline Left total hip & -1.6 & $-2.5^{*}$ & -2.6 & - \\
\hline
\end{tabular}

${ }^{*}$ indicates $Z$-score.

and 800 international units, respectively, with a recent 25hydroxyvitamin D $(25(\mathrm{OH}) \mathrm{D})$ level of $48 \mathrm{ng} / \mathrm{mL}$. At the time of her referral to our clinic, her BMD revealed a T-score of -1.6 at the lumbar spine, -2.2 at the left femoral neck, and -1.6 at the total left hip. She was then started on teriparatide $20 \mathrm{mcg}$ subcutaneously once a day. After 2 years of treatment, her BMD increased by $7.2 \%$ at the lumbar spine, but the total left hip was unchanged. She did not experience any falls or develop new fractures. No adverse effects from teriparatide therapy were reported.

Case 2. Patient 2 was a 35-year-old Caucasian female with CF (homozygous $\Delta$ F508 mutation) and CF-related diabetes who was referred to our clinic for evaluation of osteoporosis. She had been diagnosed with osteoporosis one year prior and treated with alendronate $70 \mathrm{mg}$ weekly for approximately 11 months. At the time of presentation to our clinic, she had been off bisphosphonates therapy for 2 months. Her history was significant for three fragility fractures in the past five years (two rib fractures and one sacral fracture). In addition to $1,000 \mathrm{mg}$ of calcium twice a day and 2,000 units of vitamin D daily, she had been on chronic steroids for the past two years for the treatment of allergic bronchopulmonary aspergillosis. She reported having normal puberty with menarche at age 12 and was on oral contraceptive pills containing estrogen. Her most recent serum 25(OH)D level was $56 \mathrm{ng} / \mathrm{mL}$, and her BMD demonstrated a $Z$-score of -1.6 at the lumbar spine, -1.9 at the left femoral neck, and -2.5 at the left total hip. Teriparatide $20 \mathrm{mcg}$ subcutaneously once a day was initiated and after completing two years of therapy, her BMD increased by $11 \%$ at the lumbar spine and by $10.2 \%$ at the left total hip. During the treatment period with teriparatide, she did report another rib fracture that occurred after excessive coughing. No adverse effects specific to teriparatide therapy were reported.

Case 3. Patient 3 was a 31-year-old Caucasian male with CF (2184delA/R553 mutation) referred to our clinic for evaluation of osteoporosis and vitamin D deficiency. He had been diagnosed 1 year prior with osteoporosis and was started on alendronate therapy, but he was noncompliant with treatment after one month. He was consistently taking $1,000 \mathrm{mg}$ of calcium per day and 50,000 units of vitamin D twice monthly. His most recent 25(OH)D level was $33 \mathrm{ng} / \mathrm{mL}$. He did not have a history of fractures or bone related pain; however, he had a significant decline in his hip BMD over the past 2 years. His right hip total T-score was -3.1, a $6 \%$ decrease from two years prior. He also had significant worsening of his T-score at the left hip of -2.6 , representing a decrease of $5 \%$ in two years and an overall decrease of $7 \%$ from baseline. His T-score at the lumbar spine was -2.1. Given the rapid decline in his BMD, teriparatide was initiated. After 2 years of teriparatide treatment, our patient had stabilization of his hip BMD with no further significant bone loss (despite being still in the osteoporotic range). In addition, his BMD at the lumbar spine increased by $7.6 \%$. He tolerated teriparatide therapy well with no reported adverse effects.

Case 4. Patient 4 was a 48 -year-old Caucasian female with $\mathrm{CF}$ due to R117H/R560T mutation. She was referred to our clinic for evaluation of osteopenia and a decline in her hip BMD. She was a very avid runner, running approximately 4 miles a day. She did not have any history of falls, fractures, or bone pain. She was starting to have some irregular menstrual periods and hot flashes from perimenopause. She took a multivitamin on a regular basis. Her serum 25(OH)D level upon referral to our clinic was $47 \mathrm{ng} / \mathrm{mL}$. Over the past 2 years, our patient's hip BMD had decreased approximately $4.5 \%$ with a T-score of -1.5 at the left femoral neck. Her $\mathrm{BMD}$ at the lumbar spine remained within the normal range with a T-score of -0.7 . Given the rapid decline in her BMD in 2 years, we decided to start her on teriparatide. Soon after initiating therapy, the patient self-discontinued teriparatide due to extreme nausea. She was then started on alendronate $70 \mathrm{mg}$ weekly but stopped it 1 week later due to severe myalgias. Currently she is maintained on calcium and vitamin D supplementation only.

\section{Discussion}

It is well established that bone disease is a common and major complication for individuals with CF. The prevalence of osteopenia, osteoporosis, and radiological fractures in adults with CF has been reported to be $34-38 \%, 13-27 \%$, and $19-35 \%$ [8-11]. Fracture rates have been reported to be approximately 
twofold higher in adults with CF as compared with the general population [12]. Moreover, the prevalence of bone disease in the CF population increases with age and has been correlated with severity of pulmonary disease and nutritional status $[13,14]$. As such, recommendations for screening and treatment of CF-related bone disease have been developed [15-18]. Bisphosphonates have been widely used and have been shown to be efficacious in treating bone disease in CF [19-23]. Despite the promising effects of bisphosphonates, concerns about the long-term safety and tolerability of this medication still exist $[16,24]$. Potential problems with oral bisphosphonates therapy in the CF population include poor absorption leading to ineffective therapy in some patients and concerns of higher incidence of erosive pill esophagitis [16]. Furthermore, intravenous administration of bisphosphonates has been associated with severe bone pain and flu-like symptoms in CF patients, which could adversely impact the nutritional and pulmonary status of patients with $\mathrm{CF}$ [16]. Thus, alternative treatment strategies in this high-risk population are of great interest.

Teriparatide, a recombinant form of human parathyroid hormone, has been approved as a bone formation agent for use in osteoporotic postmenopausal women $[25,26]$. This medication has been shown to increase BMD and reduce fracture rate by increasing the production of new bone via anabolic effects on osteoblasts [27]. Randomized controlled trials evaluating teriparatide and bisphosphonates therapy in postmenopausal women with osteoporosis have suggested that teriparatide is effective in increasing BMD and reducing fracture risk [28]. However, there is no data regarding the effectiveness of teriparatide in patients with CF.

In the three out of four patients who successfully completed therapy with teriparatide for CF-related bone disease, significant improvements in BMD were seen after 2 years of therapy. These findings suggest that anabolic therapy for bone disease in the CF population may be an effective and safe treatment modality, particularly in those who are intolerant of bisphosphonates. One patient was intolerant of the teriparatide but she was also intolerant of bisphosphonates. Given these promising results in these 3 patients, further studies are warranted to evaluate the efficacy and safety profile of recombinant human parathyroid hormone analogue therapy in the CF population.

\section{Conclusion}

In patients with CF-related bone disease, teriparatide may be a potential treatment option. This report highlights the need for further investigation into the use of teriparatide in the CF population.
Abbreviations
CF: $\quad$ Cystic fibrosis
BMD: $\quad$ Bone mineral density
CFTR: Cystic fibrosis transmembrane
conductance regulator
MAC: $\quad$ Mycobacterium avium complex
25(OH)D: 25-hydroxyvitamin D.

\section{Conflict of Interests}

The authors declare that there is no conflict of interests regarding the publication of this paper.

\section{References}

[1] I. Legroux-Gérot, S. Leroy, C. Prudhomme et al., "Bone loss in adults with cystic fibrosis: prevalence, associated factors, and usefulness of biological markers," Joint Bone Spine, vol. 79, no. 1, pp. 73-77, 2012.

[2] F. Flohr, A. Lutz, E. M. App, H. Matthys, and M. Reincke, "Bone mineral density and quantitative ultrasound in adults with cystic fibrosis," European Journal of Endocrinology, vol. 146, no. 4, pp. 531-536, 2002.

[3] V. Grey, S. Atkinson, D. Drury, L. Casey, G. Ferland, and C. Gundberg, "Prevalence of low bone mass and deficiencies of vitamins $\mathrm{D}$ and $\mathrm{K}$ in pediatric patients with cystic fibrosis from 3 Canadian centers," Pediatrics, vol. 122, no. 5, pp. 1014-1020, 2008.

[4] R.-M. Javier and J. Jacquot, "Bone disease in cystic fibrosis: what's new?” Joint Bone Spine, vol. 78, no. 5, pp. 445-450, 2011.

[5] S. J. King, D. J. Topliss, T. Kotsimbos et al., "Reduced bone density in cystic fibrosis: $\Delta$ F508 mutation is an independent risk factor," European Respiratory Journal, vol. 25, no. 1, pp. 54-61, 2005.

[6] L. L. Wolfenden, S. E. Judd, R. Shah, R. Sanyal, T. R. Ziegler, and V. Tangpricha, "Vitamin D and bone health in adults with cystic fibrosis," Clinical Endocrinology, vol. 69, no. 3, pp. 374-381, 2008.

[7] "International guidelines for the selection of lung transplant candidates. The American Society for Transplant Physicians (ASTP)/American Thoracic Society(ATS)/European Respiratory Society(ERS)/International Society for Heart and Lung Transplantation(ISHLT)," American Journal of Respiratory and Critical Care Medicine, vol. 158, no. 1, pp. 335-339, 1998.

[8] J. Paccou, N. Zeboulon, C. Combescure, L. Gossec, and B. Cortet, "The prevalence of osteoporosis, osteopenia, and fractures among adults with cystic fibrosis: a systematic literature review with meta-analysis," Calcified Tissue International, vol. 86, no. 1, pp. 1-7, 2010.

[9] C. S. Haworth, P. L. Selby, A. K. Webb et al., "Low bone mineral density in adults with cystic fibrosis," Thorax, vol. 54, no. 11, pp. 961-967, 1999.

[10] S. L. Elkin, A. Fairney, S. Burnett et al., "Vertebral deformities and low bone mineral density in adults with cystic fibrosis: a cross-sectional study," Osteoporosis International, vol. 12, no. 5, pp. 366-372, 2001.

[11] C. Brenckmann, A. Papaioannou, A. Freitag et al., "Osteoporosis in Canadian adult cystic fibrosis patients: a descriptive study," BMC Musculoskeletal Disorders, vol. 4, article 1, 2003.

[12] R. M. Aris, J. B. Renner, A. D. Winders et al., "Increased rate of fractures and severe kyphosis: sequelae of living into adulthood with cystic fibrosis," Annals of Internal Medicine, vol. 128, no. 3, pp. 186-193, 1998.

[13] A. S. Neri, I. Lori, G. Taccetti et al., "Alteration of bone mineral density in cystic fibrosis adults," Chest, vol. 130, no. 6, pp. 19521953, 2006.

[14] R. M. Aris, D. A. Ontjes, H. E. Buell et al., "Abnormal bone turnover in cystic fibrosis adults," Osteoporosis International, vol. 13, no. 2, pp. 151-157, 2002. 
[15] R. Aris, G. Lester, and D. Ontjes, "Treatment of bone disease in cystic fibrosis," Current Opinion in Pulmonary Medicine, vol. 10, no. 6, pp. 524-530, 2004.

[16] R. M. Aris, P. A. Merkel, L. K. Bachrach et al., "Guide to bone health and disease in cystic fibrosis," The Journal of Clinical Endocrinology \& Metabolism, vol. 90, no. 3, pp. 1888-1896, 2005.

[17] T. M. Hecker and R. M. Aris, "Management of osteoporosis in adults with cystic fibrosis," Drugs, vol. 64, no. 2, pp. 133-147, 2004.

[18] I. Sermet-Gaudelus, M. L. Bianchi, M. Garabédian et al., "European cystic fibrosis bone mineralisation guidelines," Journal of Cystic Fibrosis, vol. 10, supplement 2, pp. S16-S23, 2011.

[19] R. M. Aris, G. E. Lester, M. Caminiti et al., "Efficacy of alendronate in adults with cystic fibrosis with low bone density," American Journal of Respiratory and Critical Care Medicine, vol. 169, no. 1, pp. 77-82, 2004.

[20] S. P. Conway, B. Oldroyd, A. Morton, J. G. Truscott, and D. G. Peckham, "Effect of oral bisphosphonates on bone mineral density and body composition in adult patients with cystic fibrosis: a pilot study," Thorax, vol. 59, no. 8, pp. 699-703, 2004.

[21] R. M. Aris, G. E. Lester, J. B. Renner et al., "Efficacy of pamidronate for osteoporosis in patients with cystic fibrosis following lung transplantation," American Journal of Respiratory and Critical Care Medicine, vol. 162, no. 3, pp. 941-946, 2000.

[22] C. S. Haworth, P. L. Selby, J. E. Adams, E. B. Mawer, A. W. Horrocks, and A. K. Webb, "Effect of intravenous pamidronate on bone mineral density in adults with cystic fibrosis," Thorax, vol. 56, no. 4, pp. 314-316, 2001.

[23] C. S. Haworth, L. Sharples, V. Hughes et al., "Multicentre trial of weekly risedronate on bone density in adults with cystic fibrosis," Journal of Cystic Fibrosis, vol. 10, no. 6, pp. 470-476, 2011.

[24] C. S. Haworth, P. L. Selby, A. K. Webb, E. B. Mawer, J. E. Adams, and T. J. Freemont, "Severe bone pain after intravenous pamidronate in adult patients with cystic fibrosis," The Lancet, vol. 352 , no. 9142, pp. 1753-1754, 1998.

[25] V. Z. Borba and N. C. Mañas, "The use of PTH in the treatment of osteoporosis," Arquivos Brasileiros de Endocrinologia e Metabologia, vol. 54, no. 2, pp. 213-219, 2010.

[26] R. M. Neer, C. D. Arnaud, J. R. Zanchetta et al., "Effect of parathyroid hormone (1-34) on fractures and bone mineral density in postmenopausal women with osteoporosis," The New England Journal of Medicine, vol. 344, no. 19, pp. 1434-1441, 2001.

[27] E. Canalis, A. Giustina, and J. P. Bilezikian, "Mechanisms of anabolic therapies for osteoporosis," The New England Journal of Medicine, vol. 357, no. 9, pp. 905-916, 2007.

[28] J.-J. Body, G. A. Gaich, W. H. Scheele et al., "A randomized double-blind trial to compare the efficacy of teriparatide [recombinant human parathyroid hormone (1-34)] with alendronate in postmenopausal women with osteoporosis," The Journal of Clinical Endocrinology \& Metabolism, vol. 87, no. 10, pp. 4528-4535, 2002. 


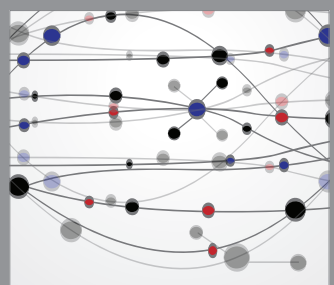

The Scientific World Journal
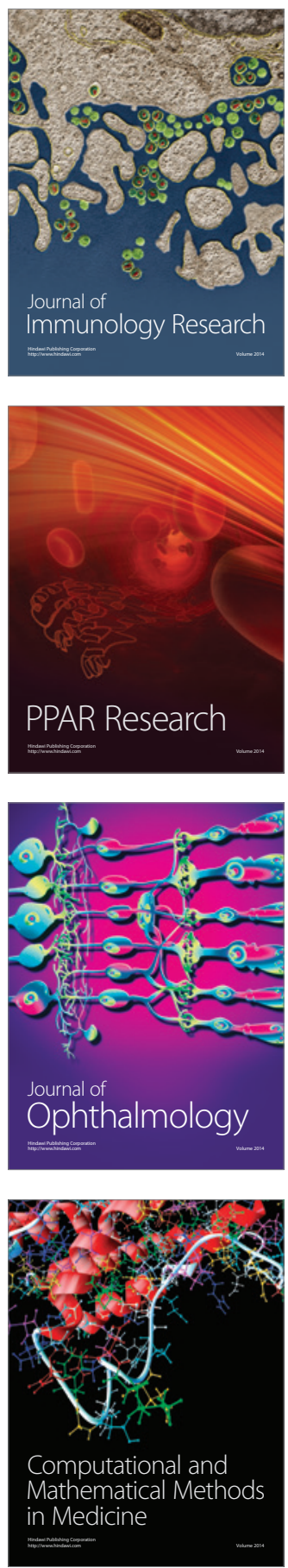

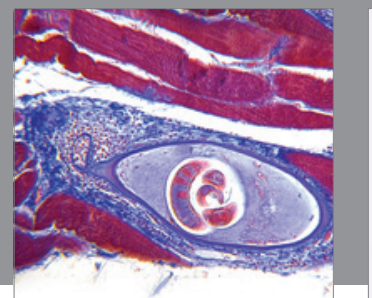

Gastroenterology

Research and Practice
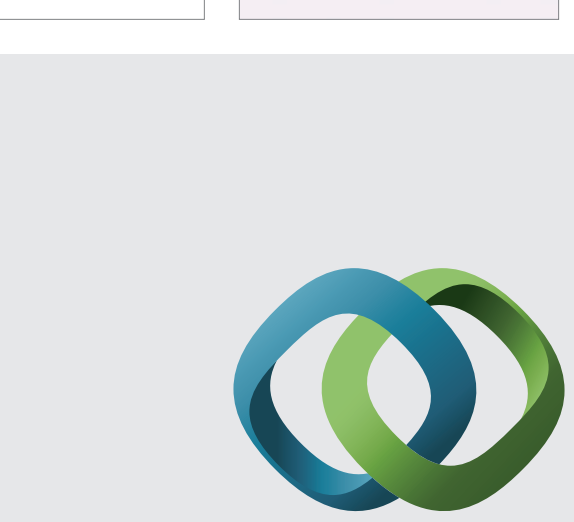

\section{Hindawi}

Submit your manuscripts at

http://www.hindawi.com
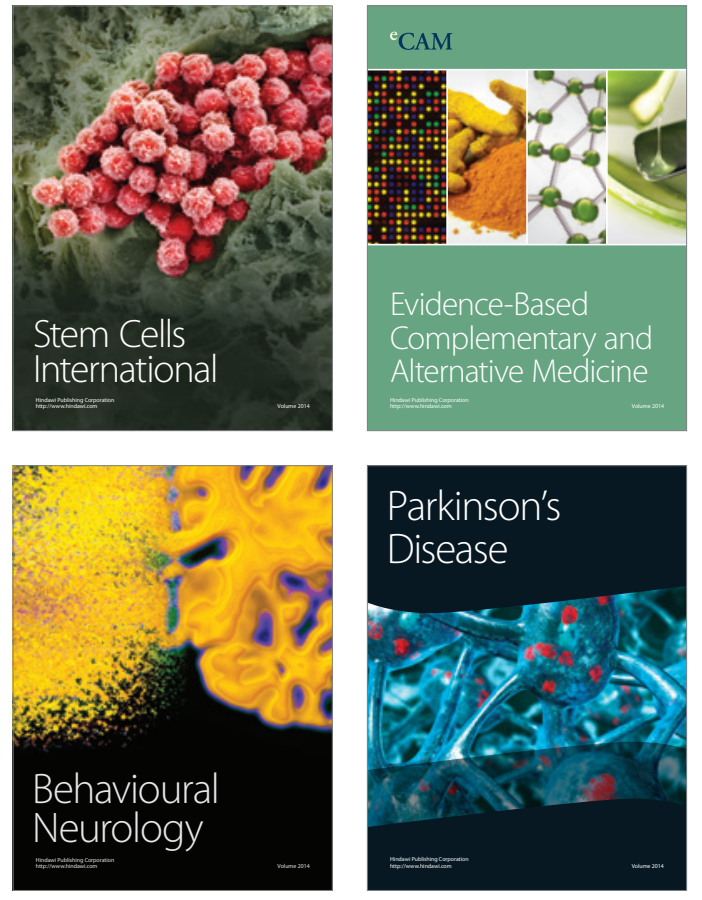
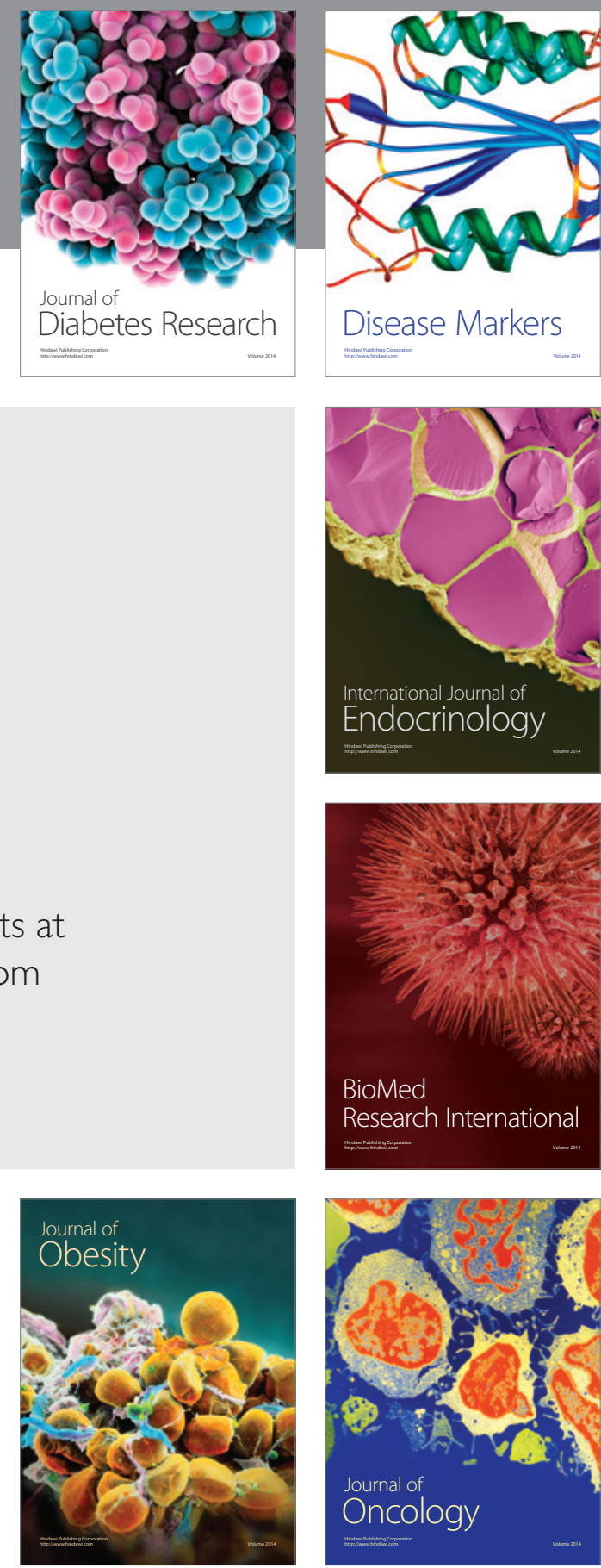

Disease Markers
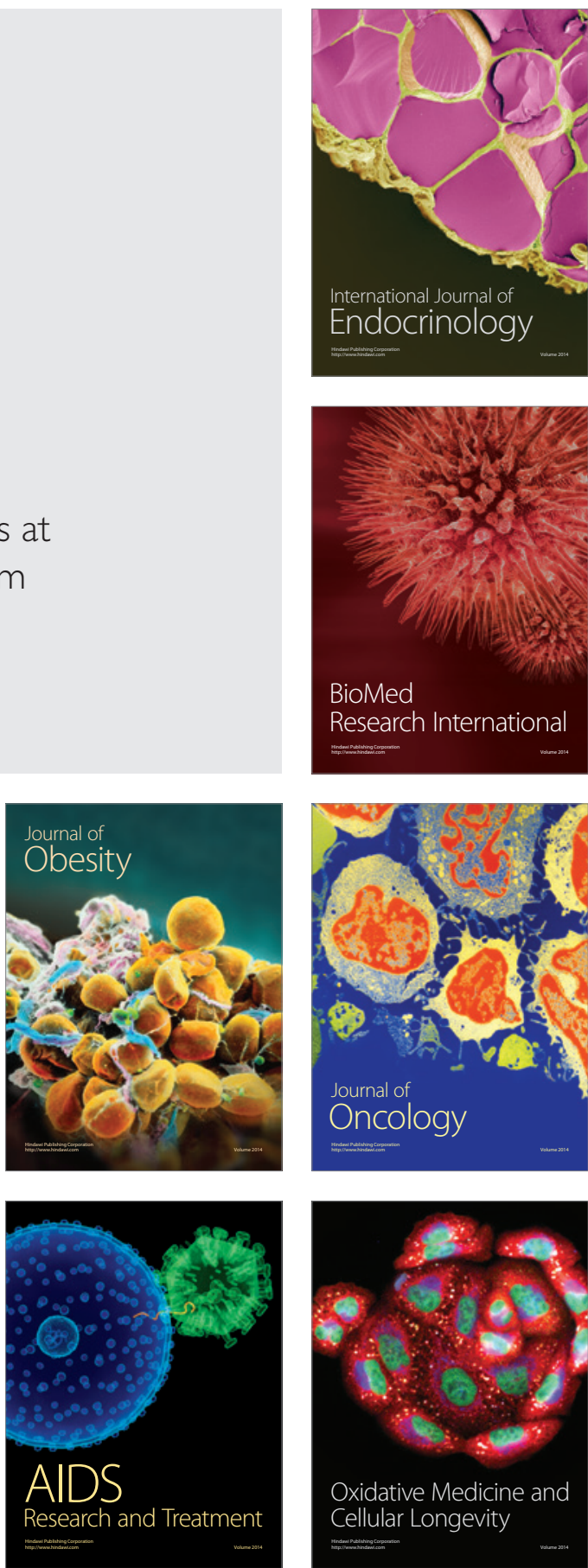